\title{
A whiter shade of pale, a blacker shade of dark: Parameters of spatially induced blackness
}

\author{
DAVID L. BIMLER, ${ }^{1}$ GALINA V. PARAMEI, ${ }^{2}$ AND CHINGIS A. IZMAILOV ${ }^{3}$ \\ ${ }^{1}$ Department of Health and Human Development, Massey University, New Zealand \\ ${ }^{2}$ Institute of Psychology, Darmstadt University of Technology, Germany \\ ${ }^{3}$ Department of Psychophysiology, Moscow Lomonosov State University, Moscow, Russia
}

(Received November 8, 2005; AcCePted November 29, 2005)

\begin{abstract}
The surface-mode property of "blackness" is induced by simultaneous contrast with an adjacent, more luminant surround. As numerous studies have shown, the degree of blackness induced within an achromatic test field is a function of the relative luminance of the adjacent chromatic inducing field, but not of its hue. But in the converse case of chromatic test fields, susceptibility to blackening has been reported to vary with wavelength. The present study investigates this possibility, that some wavelengths are more susceptible. We also questioned whether "white" and "black" sensory components function as opposites in blackness appearance. We recorded the appearance of a central monochromatic test field of constant luminance $\left(10 \mathrm{~cd} / \mathrm{m}^{2}\right)$, with wavelength ranging across the visible spectrum, while a broadband white annulus was set to six luminance levels ranging across three log steps. Three color-normal observers followed a color-naming technique. All six opponent-hue names and their combinations were response options; blackness and whiteness in the test field could therefore be reported independently. Of primary interest were the achromatic responses. When represented within a multidimensional space, these revealed the "white-to-black" dimension but in addition a quality (dimension) of "desaturation." Compared against chromatic properties of the test field, the results provide evidence that blackness is a function of inducing field brightness (not luminance). This result is in accord with observations made by Shinomori et al. (1997) using a different procedure. We conclude that blackness induction occurs at a stage of visual processing subsequent to the origin of the brightness signal from a combination of opponent-process channels.
\end{abstract}

Keywords: Spatially induced blackness, Chromatic light, Color naming, Bidimensional achromatic quality

\section{Introduction}

In the language of color description, "red" and "green" form the polar extremes of one dimension of color experience; they are not used together to describe the same hue. In the same way, "blue" and "yellow" constitute the poles of a second chromatic dimension. "White" and "black" are a third pair of polar opposites, but their behavior differs in certain ways from the previous pairs. First, they can coexist, so that a given shade of gray might be described as containing black and white in equal amounts. Second, "blackness" is not observed in isolation, and cannot be examined in the simplified situation of "aperture mode." Blackness only exists as part of an environment, and must be induced by contrast with a lighter color elsewhere in the visual field (spatial contrast) or immediately preceding the test stimulus (temporal contrast).

Address correspondence and reprint requests to: David L. Bimler, Department of Health and Human Development, Massey University, Private Bag 11-222, Palmerston North, New Zealand. E-mail: d.bimler@ massey.ac.nz
A number of studies have examined blackness induction into a white center by a chromatic annulus with wavelength $\lambda_{\mathrm{A}}$. Shinomori et al. (1994) elicited judgments of blackness and whiteness while varying the relative annulus luminance; the curves of "percentage blackness" followed the same shape at each $\lambda_{\mathrm{A}}$. Other studies elicited the annulus luminance $L_{\mathrm{A}}$ required to induce a given level of blackness, as a function of $\lambda_{\mathrm{A}}$. Criteria included "perceptible greyness" (Evans, 1967), just-noticeable blackness (Mount \& Thomas, 1968), and complete blackness and the disappearance of any contour (e.g. Werner et al., 1984). These are all criteria of decreased subjective lightness. Kulp and Fuld (1989) found that the variables involved in the phenomenon are the same whichever criterion is used: there is a consensus that blackness induction into an achromatic center depends only on the ratio of luminance between the contrasting elements. Changes in annulus $\lambda_{\mathrm{A}}$ influence $L_{\mathrm{A}}$ through the luminance function $V_{\lambda}$, but they have no effect otherwise. Like $V_{\lambda}$, the blackness-induction function for a white center proves to be additive when wavelengths are combined in the annulus (Volbrecht et al., 1990).

It is tempting to assume that the same principle governs the opposite situation where blackness is induced into a chromatic 
center (wavelength $\lambda_{\mathrm{C}}$ ) by a white surround. One objective of this study is to investigate the converse possibility, that the role of color in the interaction is not symmetric, with $\lambda_{\mathrm{C}}$ as an independent parameter of blackness induction (Fuld et al., 1986). This would have implications for the stage of color processing where contrast is computed.

Cicerone et al. (1986) and Mount and Thomas (1968) both examined four settings of $\lambda_{\mathrm{C}}$ while varying annulus wavelength $\lambda_{\mathrm{A}}$. The published details do not permit comparison among $\lambda_{\mathrm{C}}$ settings to see whether $\lambda_{\mathrm{C}}$ has any independent role in induction (e.g. through variations in "chromatic strength"). However, the efficiency of blackness induction as a function of $\lambda_{\mathrm{A}}$ retained the characteristic shape of $V_{\lambda}$, ruling out any interaction between $\lambda_{\mathrm{A}}$ and $\lambda_{\mathrm{C}}$.

Evans and Swenholt (1967) examined two criteria as a function of $\lambda_{\mathrm{C}}$ : the chromatic threshold between complete central blackness and the last vestige of color, and $G_{0}$, chromatic strength, defined as the threshold of perceptible desaturation by the first appearance of gray. The two functions were parallel, separated by about a log step in annulus:center luminance ratio. The value $G_{0}$ departed from the luminance function $V_{\lambda}$ and bore a closer resemblance to the "purity discrimination" function (or "absolute saturation threshold").

Shinomori et al. (1997) obtained strikingly similar results. The annulus:center luminance ratio required for complete central blackness was higher for some wavelengths than others, with the log ratio varying from 2.5 to 4 . Plotted against $\lambda_{\mathrm{C}}$, the function closely resembled the brightness function $B_{\lambda}$. Like $B_{\lambda}$, but unlike $V_{\lambda}$, blackness induction departed from additive behavior when the center contained combinations of wavelengths.

The present study used an extended color-naming procedure to elicit descriptions of the appearance of monochromatic stimuli in the presence of luminance contrast. Observers' responses were analyzed to extract an index of spatially induced blackness. We consider a wider range of intensity ratios than Shinomori et al. (1997): the broadband white annulus (2850 K), providing the contrast, ranged in luminance in six levels across three orders of magnitude, from $1 / 50$ to 20 times the intensity of the central stimulus.

Fuld and Otto (1985) collected color-naming responses for the same situation of a monochromatic central stimulus (wavelength $\lambda_{\mathrm{C}}$ ) darkened by contrast. However, they studied relatively few wavelengths, and did not examine the possibility that $\lambda_{\mathrm{C}}$ was a parameter in the spatial induction of blackness.

A secondary objective of the present study is to investigate the dimensionality underlying the achromatic responses, that is, how many independent qualities they convey. Clearly the two terms black and white can operate as the opposite extremes of a bipolar continuum of subjective lightness. In the color-naming data of Fuld and Otto (1985), subjects hardly ever reported both within the same stimulus. However, "black" and "white" both convey a quality of desaturation, and they can be used together to characterize a grayness or lack of hue. Quinn et al. (1985) and Fuld et al. (1986) found that the two terms can indeed coexist. Thus it is not clear how far they function as antagonists. Heggelund (1992) argues that the true opposition is between black and luminous, defining one axis between them, while whiteness is a separate, orthogonal axis. Again, "whiteness" and "blackness" are separate processes in the hyperspherical model of color discrimination (Izmailov, 1981; Izmailov \& Sokolov, 1991). It would seem that a color-naming response of "white" can arise for two different reasons.

\section{Materials and Method}

The experiment was carried out at Moscow Lomonosov State University, Russia.

Subjects were three Russian-speaking women aged 21, 22, and 24 years. They were confirmed as normal trichromats using the Rautian anomaloscope (Rautian, 1957).

Stimuli: 156 stimuli were presented foveally in Maxwellian view. Each consisted of a monochromatic center of fixed luminance $\left(L_{\mathrm{C}}=10 \mathrm{~cd} / \mathrm{m}^{2}\right)$ subtending $2 \mathrm{deg}$, and a contrast-inducing annulus with 2 deg inner and 6 deg outer diameter. The central wavelength $\lambda_{\mathrm{C}}$ ranged from 425 to $675 \mathrm{~nm}$, with 25 values provided by interference filters (with 4 to $6 \mathrm{~nm}$ half-bandwidth), plus a broadband tungsten white light (ca. $2850 \mathrm{~K}$ ). Six levels of annulus luminance $L_{\mathrm{A}}$ were tested, ranging across three orders of magnitude: $0.2,2,10,20,100$, and $200 \mathrm{~cd} / \mathrm{m}^{2}$ (i.e. values of the annulus:center luminance ratio were $0.02,0.2,1,2,10,20$ ).

Procedure: Each stimulus was presented 20 times over 10 sessions, in pseudo-random order. Subjects described the appearance of the center with one, two, or three terms, in order of decreasing salience. The six permitted terms were the Russian equivalents of red, yellow, green, blue (sinij), white, and black ( $\mathrm{R}$, $\mathrm{Y}, \mathrm{G}, \mathrm{B}, \mathrm{W}$, and $\mathrm{Bk}$ ).

Analysis: A color term scored 10 points if it was used in isolation; two terms received 6 and 4 points in order of salience; three terms received 5, 3, and 2 points. Points for each term were summed over presentations, giving a value (from 0 to 200) for that color-naming function. Responses across the subjects were sufficiently similar to combine them.

The similarity between any pair of stimuli was quantified as the covariance between the corresponding vectors of color-name values, before analyzing the matrix of similarities with nonmetric multidimensional scaling (MDS). MDS represents stimuli as points in an $n$-dimensional space, arranged so that interpoint distances reflect interitem similarities. A badness-of-fit function Stress $_{1}$ quantifies the mismatch between distances and similarities.

\section{Results and discussion}

Solutions with two to five dimensions were fitted to the 156-by156 covariance matrix. Respective values of Stress $_{1}$ were $19.2 \%$, $8.8 \%, 6.0 \%$, and $4.8 \%$. We argue that the last incremental dimension to provide a substantial improvement in fit is the fourth; that is, the solution is four-dimensional (cf. Izmailov et al., 1999). Plausible interpretations can be assigned to the four axes (labeled as $D 1, D 2, D 3, D 4)$ but not to a fifth. The solution consists of 156 points, each located by coordinates $\left\{x_{i 1}, x_{i 2}, x_{i 3}, x_{i 4}\right\}$.

Fig. 1 plots the coordinates of the points against $\lambda_{\mathrm{C}}$. Here $D 1$ and $D 2$ can be identified as perceptual chromatic axes. They are almost identical to R-G and B-Y difference functions. Two achromatic axes D3 and D4 contain all the achromatic content of the color-naming descriptions. The solution is rotated so that D3 maximizes the dispersal of the six broadband-center stimuli, arranging them along a gradient from lightest to darkest annulus. This prompts an interpretation of D3 as "strength of blackness from contrast induction." Axis D4 is interpreted as "desaturation," since $x_{i 4}$ values for chromatic stimuli coincide with direct ratings of desaturation (Gordon \& Abramov, 1988), while the broadband stimuli are all at the positive extreme. Axis $D 4$ further agrees with the desaturation axis found in an earlier MDS analysis of similar data (Bimler \& Paramei, 2005).

It is clear from Fig. 1c that when the ratio $L_{\mathrm{A}} / L_{\mathrm{C}}$ between annulus and center luminance is sufficiently high, induced black- 

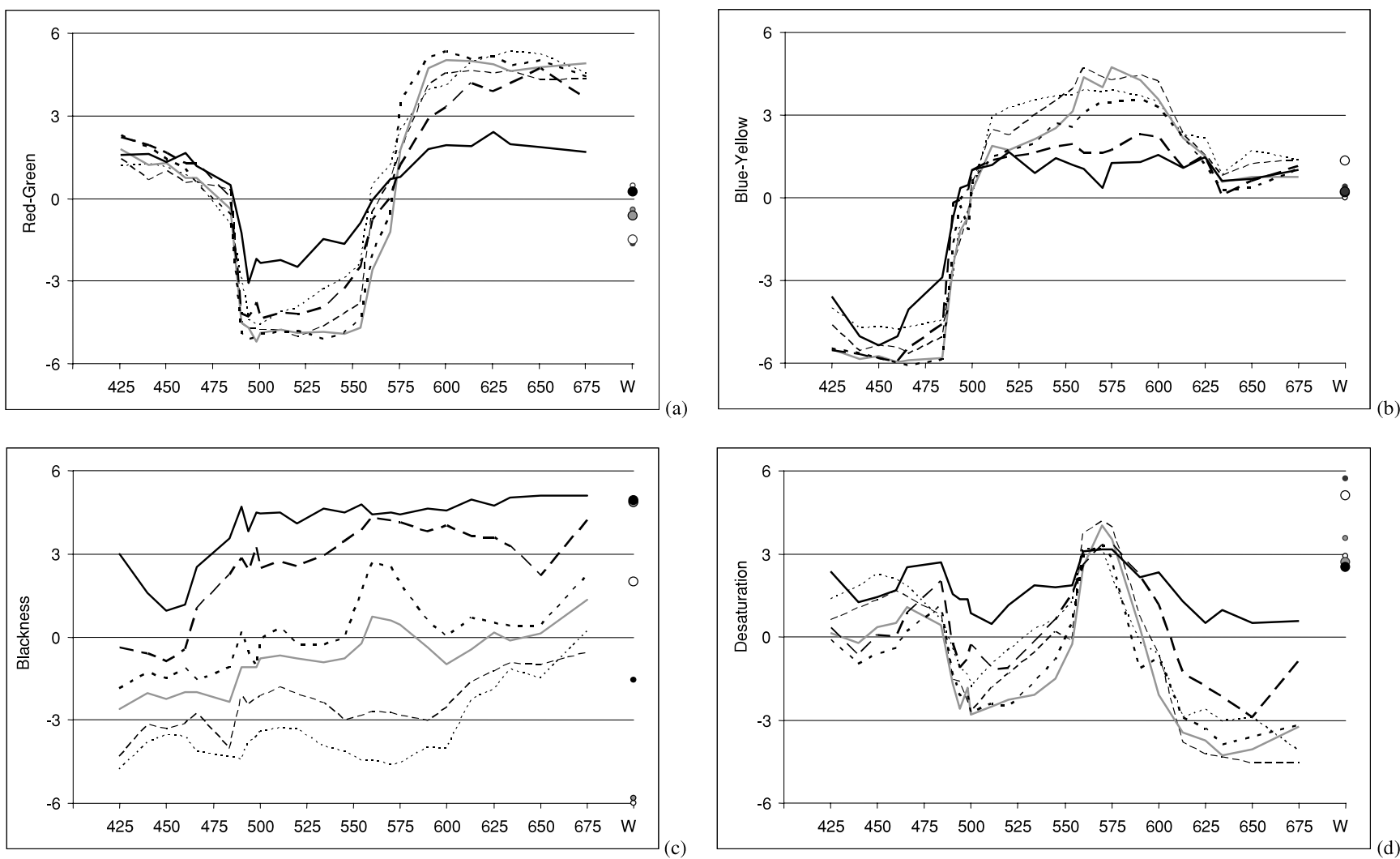

Fig. 1. Coordinates of color points in the $4 \mathrm{D}$ color space (vertical axis, arbitrary units) plotted against central wavelength $\lambda_{\mathrm{C}}(\mathrm{nm})$ : (a) $x_{i 1}$ ("green/red"); (b) $x_{i 2}$ ("blue/yellow"); (c) $x_{i 3}$ ("blackness induction"); (d) $x_{i 4}$ ("desaturation"). Lines connect stimuli with same annulus luminance $\left(L_{\mathrm{A}}\right)$, except for white centers at the right.

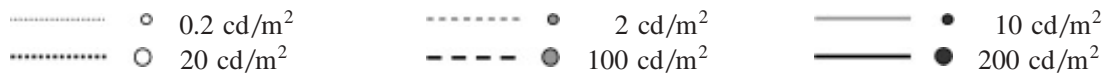

ness dominates the center whatever its wavelength $\lambda_{\mathrm{C}}$. That is, $x_{i 3}$ reaches a ceiling value. But at lower ratios $\left(L_{\mathrm{A}} / L_{\mathrm{C}} \leq 1\right)$ the impact of a step increase in $L_{\mathrm{A}}$ varies across $\lambda_{\mathrm{C}}$. For some wavelengths (e.g. yellow centers), $x_{i 3}$ increases disproportionately, and the corresponding line in Fig. 1c is displaced upward. Less-responsive wavelengths catch up as the annulus luminance continues to increase.

For a given $\lambda_{\mathrm{C}}$, across the six luminance ratios $L_{\mathrm{A}} / L_{\mathrm{C}}, x_{i 3}$ values approximate a sigmoid function. The primary research question is addressed by finding the inverse to the sigmoid function for each $\lambda_{\mathrm{C}}$. The level of luminance contrast required to produce some chosen value of $x_{i 3}$-that is, to induce a given criterion of blackness content-can then be calculated as a function of central wavelength $\lambda_{\mathrm{C}}$. We do this by interpolating within a logistic curve, fitted to the $x_{i 3}$ values for the six distinct values of $L_{\mathrm{A}} / L_{\mathrm{C}}$. Results are shown in Fig. 2.

The MDS solution is still open to arbitrary rotations of the achromatic plane. It might be preferable, for instance, to rotate it through 45 deg and create separate achromatic axes of "brightness" and "darkness," but the alignment used here is most convenient for addressing the research questions. Note that distance from the origin is nearly constant across the points, where this distance is $R_{i}=\sqrt{\left(x_{i 1}^{2}+x_{i 2}^{2}+x_{i 3}^{2}+x_{i 4}^{2}\right)}$. If the solution is rescaled so that mean $\left(R_{i}\right)=1$, then the standard deviation of $R_{i}$ is 0.016 . Points do not fill the $4 \mathrm{D}$ space, but are roughly confined to a locally three-dimensional "surface." The solution is essentially a hyperspherical shell: it remains compatible with Izmailov and Sokolov's (1991) hyperspherical model of color discrimination, and with their hyperspherical coordinate system.

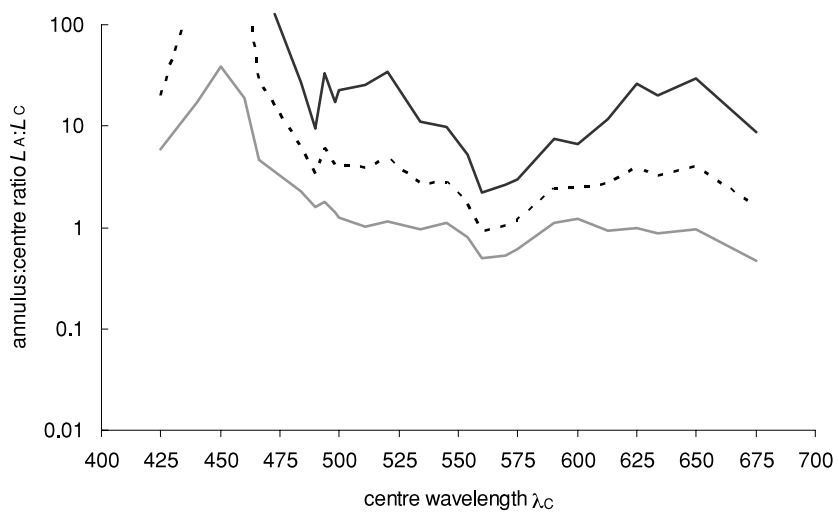

Fig. 2. Luminance ratio $L_{\mathrm{A}}: L_{\mathrm{C}}$ predicted to produce three particular values of $x_{i 3}$ (i.e. to induce three specific levels of blackness induction), as a function of central wavelength $\lambda_{\mathrm{C}}$. Here $L_{\mathrm{A}}, L_{\mathrm{C}}$ are luminance of annulus and chromatic center. Solid grey, broken, and solid black lines indicate $x_{i 3}=0,2,4$. 


\section{Conclusions}

The curves of Fig. 2 are gratifyingly close to previous measurements of blackness induction into monochromatic centers (e.g. Shinomori et al., 1997, Fig. 3), and of the visible-grayness or $G_{0}$ criterion (Evans \& Swenholt, 1967, Fig. 3). The 570-nm trough in resistance to blackness induction, the smaller dip at $490 \mathrm{~nm}$, and the peaks at 520 and $650 \mathrm{~nm}$ are all familiar features from those counterparts. The declines in the curves at the highest and lowest wavelengths could be artefacts, but they are also present for some subjects in the results of Shinomori et al.

The wavelength dependence of blackness induction (its efficiency or action spectrum) is very similar to the brightness spectrum $B_{\lambda}$, so a parsimonious explanation would involve the latter. The brightness signal is thought to arise from a nonlinear combination of opponent signals. The implication is that the blacknessinduction aspect of contrast is computed subsequently to the origin of $B_{\lambda}$, quite late in visual processing.

But less-bright wavelengths such as $570 \mathrm{~nm}$ are also low in "chrominance" or "chromatic strength": that is, a comparatively high proportion of 570-nm light is required to produce a perceptible hue when mixed with white light. Thus an alternative explanation for these results is that induced blackness is independent of $\lambda_{\mathrm{C}}$, but the blackness then dilutes or drowns out low-chrominance center wavelengths to the point of invisibility (complete blackness) more easily than high-chrominance $\lambda_{\mathrm{C}}$. In this explanation, $x_{i 3}$ is interpreted as the ratio of blackness induction to chrominance, rather than as blackness in isolation.

However, that account is excluded by Fuld et al. (1986), who used a criterion of "equal induction of black and white," where hue strength and visibility are irrelevant. Their results were similar to our Fig. 2: black and white canceled or balanced when the annulus matched the center for brightness (not luminance). This is also visible in our color-naming data for the case $L_{\mathrm{A}}=L_{\mathrm{C}}$, where the same annulus induced significant Bk-naming for less-bright yellow centers, and significant W-naming for high-brightness blue centers. This can be seen in the $L_{\mathrm{A}}=10 \mathrm{~cd} / \mathrm{m}^{2}$ line of Fig. 1c, and in the lowest curve of Fig. 2. We are left with the direct link between blackness induction and brightness.

In an earlier study of monochromatic, aperture-mode stimuli (Bimler \& Paramei, 2005), where Bk was not among the response categories in the color-naming data, the third dimension of a MDS solution was identified as "desaturation." It is replicated by the fourth axis of the present 4D solution. Thus the orthogonal dimensions $D 3$ and $D 4$ both have plausible interpretations, confirming that neither is an artefact. As noted above, "white" can also convey implications of both lightness and desaturation. There is a further implication that $\mathrm{Bk}$ is not simply the inverse or absence of $\mathrm{W}$ : between them, the two naming functions capture more than a single aspect of color appearance. A number of wavelength/ luminance-ratio conditions required a combination of black and white for a description. This is not simply the result of summing data across three subjects with different response functions, since it holds true when individual subjects' responses are examined.

\section{Acknowledgments}

Preparation of the article by G.V.P. was supported by a fellowship from the Hanse Institute for Advanced Study, Germany. We are grateful to J. Werner for helpful comments on an earlier version of the paper.

\section{References}

Bimler, D.L. \& Paramei, G.V. (2005). Bezold-Brücke effect in normal trichromats and protanopes. Journal of the Optical Society of America A 22, 2120-2136.

Cicerone, C.M., Volbrecht, V., Donnelly, S.K. \& Werner, J.S. (1986). Perception of blackness. Journal of the Optical Society of America A 3, 432-436.

Evans, R.M. (1967). Luminance and induced colors from adaptation to 100-millilambert monochromatic light. Journal of the Optical Society of America 57, 279-281.

Evans, R.M. \& Swenholt, B.K. (1967). Chromatic strength of colors: Dominant wavelength and purity. Journal of the Optical Society of America 57, 1319-1324.

Fuld, K. \& OтTо, T.A. (1985). Colors of monochromatic lights that vary in contrast-induced brightness. Journal of the Optical Society of America A 2, 76-83.

Fuld, K., Отto, T.A. \& Slade, C.W. (1986). Spectral responsivity of the white-black channel. Journal of the Optical Society of America A 3, 1182-1188.

Gordon, J. \& Abramov, I. (1988). Scaling procedures for specifying color appearance. Color Research and Application 13, 146-152.

HegGelund, P. (1992). A bidimensional theory of achromatic color vision. Vision Research 32, 2107-2119.

IzmaILov, C.A. (1981). [Multidimensional scaling of achromatic component of color.] In Normative and Descriptive Models of Decision Making, eds. Lomov, B.F., LucE, R.D., Estes, W.K., KRYLov, V.J. \& Krylova, N.V., pp. 98-110. Moscow: Nauka (in Russian).

Izmailov, C.A. \& Sokolov, E.N. (1991). Spherical model of color and brightness discrimination. Psychological Science 2, 249-259.

Izmailov, C.A., Sokolov, E.N. \& Shtiui, S. (1999). [Spherical model of color discrimination under the conditions of simultaneous color contrast]. Vestnik Mosk. un-ta. Ser. 14. Psikhologiya No. 4, 21-36 (in Russian).

Kulp, T.D. \& Fuld, K. (1989). Black spectral responsivity. Journal of the Optical Society of America A 6, 1233-1238.

Mount, G.E. \& Thomas, J.P. (1968). Relation of spatially induced brightness changes to test and inducing wavelengths. Journal of the Optical Society of America 58, 23-27.

Quinn, P.C., Wooten, B.R. \& Ludman, E.J. (1985). Achromatic color categories. Perception and Psychophysics 37, 198-204.

Rautian, G.N. (1957). [New anomaloscope]. Biofizika 2, 734-742 (in Russian).

Shinomori, K., NaKano, Y. \& Uchikawa, K. (1994). Influence of the illuminance and spectral composition of surround fields on spatially induced blackness. Journal of the Optical Society of America A 11, 2383-2388.

Shinomori, K., Schefrin, B.E. \& Werner, J.S. (1997). Spectral mechanisms of spatially induced blackness: Data and quantitative model. Journal of the Optical Society of America A 14, 372-387.

Volbrecht, V., Werner, J.S. \& Cicerone, C.M. (1990). Additivity of spatially induced blackness. Journal of the Optical Society of America A 7, 106-112.

Werner, J.S., Cicerone, C.M., Kliegl, R. \& DellaRosa, D. (1984). Spectral efficiency of blackness induction. Journal of the Optical Society of America A 1, 981-986. 
A whiter shade of pale, a blacker shade of dark: Parameters of spatially induced blackness

Bimler, David L.

2006

http://hdl.handle.net/10179/9669

22/04/2023 - Downloaded from MASSEY RESEARCH ONLINE 